\title{
Inertial Particle Dispersion in the Lagrangian Wake of a Square Cylinder
}

\author{
G. B. Jacobs, *and K. Armstrong ${ }^{\dagger}$ \\ Department of Aerospace Engineering $\&$ Engineering Mechanics, \\ San Diego State University, San Diego, CA, 92182.
}

\begin{abstract}
A numerical investigation is presented into the relation between finite sized, inertial particle transport and entrainment in the vortex-dominated wake of a square cylinder placed in a channel at two blockage ratios and Lagrangian Coherent Structures (LCS) identified with recent Lagrangian visualization techniques. The square cylinder flow is computed with the incompressible, viscous flow solver in Fluent at a Reynolds number of 150 ensuring a periodic Karman street in the cylinder's wake. The traces of fluid and inertial particle are determined with Fluent's Discrete Phase Model, that models finite sized particles as points. The MANGEN software is used to determine contours of the Finite Time Lyapunov Exponent (FTLE) in the fluid particle flow using time-dependent flow field data computed with Fluent as input. Fluid particle transport barriers or Lagrangian Coherent Structures that repel fluid particles as visualized by maxima in the FTLE contours determined in forward-time form lobes in front of the cylinder that entrain fluid particles into the near wake behind the square. Inertial particles having a delayed response to the fluid do not entrain into the near wake for particle response times greater than ten percent of the characteristic flow time. Inertial particles closely follow the attracting structures as visualized by the FTLE in backward time up to a Stokes number of unity, when particles focus along the transport barriers. When the particle response time is comparable to the shedding period, particles are entrained in the far wake between large coherent vortex structures. With increased blockage, transport barriers form of the walls that are convected downstream along the wall and interact with the structures in the wake of the cylinder. The fluid and inertial particles closely following the transport barriers show an enhanced mixing with increased blockage.
\end{abstract}

\section{Introduction}

The dispersion of droplets and particles in unsteady flows has been studied extensively. In Crowe et al. ${ }^{4}$ the dispersion characteristics of inertial particles in a developing shear layer were categorized based on the Stokes number. The Stokes number, $S t=t_{p} / t_{f}$, is defined by the ratio of the response time of the particle, $t_{p}=\rho_{p} d_{p}^{2} / 18 \mu$, to a characteristic carrier flow time, $t_{f}=L_{f} / U_{f} . \rho_{p}$ and $d_{p}$ are the particle density and diameter, respectively. $\mu$ is the kinematic viscosity and $L_{f}$ and $U_{f}$ represent a reference length scale and velocity in the flow. Light and small particles with $S t \ll 1$ disperse like fluid particles while larger and heavier particles with $S t \gg 1$ remain largely unaffected by the flow. Intermediate-sized particles with $S t \simeq 1$ are radially centrifuged by large flow structures. In simulations of a particle-laden wake flow characterized by large scale vortices Tang et al. ${ }^{5}$ had similar findings. They observed that the particles with $S t \simeq 1$ align at the periphery of the vortices, a phenomenon termed "particle focusing".

Droplet- and particle-laden flows frequently interact with a bluff geometry. In liquid-fuel spray combustors, for example, fuel droplets can interact with a flame holder in the combustor. The particle-laden flow field over a blunt body is characterized by a recirculation region in the wake of the body that entrains particles and large-scale vortex structures in the wake behind the body that further disperse particles. Although the blunt body flow has been subject of many investigations (e.g. see Ref. 6 for a review) the particle-laden

\footnotetext{
*Assistant Professor, AIAA member, Email: gjacobs@mail.sdsu.edu

${ }^{\dagger}$ Graduate Student
} 
flow studies are somewhat limited. Brandon and Aggarwal ${ }^{7}$ simulated an incompressible flow over a square cylinder confined in a duct and analyzed the particle transport for a range of particle masses and sizes. Jacobs et. $a l^{8}$ considered the compressible flow over an unconfined square cylinder. Both of these studies confirmed the particle dispersion behaviors described above. Jacobs et al. ${ }^{8}$ further found that particles with a response time comparable to the flow shedding time period, accumulate ahead of the vortices that are convected downstream in the von Karman street in the wake of the square.

Since particle dispersion is clearly influenced by the large scale flow structures, defining and visualizing the location and boundary of these structures is an integral part to the analysis of particle dynamics. Structures and vortices in the carrier flows are traditionally identified by visualizations in the Eulerian framework, e.g instantaneous streamlines, pressure, velocity, vorticity and helicity contours. In recent years, however, analysis and visualization of structures in the Lagrangian frame moving with fluid particles has emerged.

Haller ${ }^{1}$ introduced a Lagrangian analysis that identified finite time material lines by determining the Finite Time Lyapunov Exponent (FTLE) in a velocity field. The FTLE measures the exponential divergence of neighboring particle trajectories in the flow. Since the kinematic fluid particle tracing is linear, the FTLE may be determined in a forward or a backward time direction. In forward time the FTLE measures the amount of "stretching" of the fluid, whereas in backward time the FTLE measure the amount of "contraction" of the fluid.

Maxima of the FTLE contours identify transport barriers in the fluid across which fluid particles cannot cross. Depending upon whether the maxima are in the FTLE contours determined in backward or forward time, the barrier attracts or repels fluid particles, respectively. Combinations of these barriers identify Lagrangian Coherent Structures (LCS). In Haller, ${ }^{3}$ a combination of intersecting an attracting and repelling transport barrier enclosing a circulating fluid was shown to be an excellent identifier of a vortex. The deformation of these FTLE enclosures over time are instrumental in identifying coherent structures ${ }^{9}$ and fluid entrainment mechanisms in a time-dependent flow ${ }^{10}$.

Here, we aim to determine whether the FTLE can aid in identifying the entrainment and transport mechanisms of inertial particles. To this end, we revisit the particle-laden flow over a square cylinder placed in a channel studied in Refs. 7,8. We compute the particle-laden, periodically shedding flow over the square cylinder at a Reynolds number of $R e=150$ based on the cylinder width and the bulk inflow velocity. The flow structure development near the square and the Karman vortex street that forms in the wake of the cylinder featuring clean coherent structures, set the ideal stage to study finite sized, inertial particle dispersion and entrainment. We also study the effect of two different blockages of the square cylinder in a channel on the LCS development and particle transport. We find Lagrangian Coherent structures as identified by the FTLE contours are an excellent predictor of inertial particle transport in wake flows.

The paper is organized as follows: In Section II we briefly discuss the Fluent software and models we have used to compute the flow followed by a discussion of MANGEN, a software we used to determine the Lagrangian coherent structures. We then present the computational setup. In Section ?? we discuss the inertial particle dispersions in the wake of a square cylinder. The final section (Section IV) is reserved for concluding remarks.

\section{Formulation}

We performed the computations of the particle-laden flow over a square cylinder using Fluent. The FTLE contours were determined with MANGEN, a software developed by Dr. Francois Lekien at CalTech. We first briefly summarize the models we used in this softwares, and then present the computational geometry and setup.

\section{A. Particle-Laden Flow Computation with Fluent}

The computations are performed with Fluent Inc.'s viscous, incompressible flow solver based on an implicit, finite volume discretization of second order accuracy in space and time of the Navier-Stokes flow equations. We solve the flow equation in non-dimensional form, using the cylinder width, $L_{r} e f$, and the average inflow velocity, $U_{r}$ ef to non-dimensionalize the equations.

The particle dynamics are computed using Fluent's Discrete Phase Model (DPM). In DPM, individual particles are modeled as point particles and are traced along their path in the Lagrangian frame using the kinematic equation for the particle position: 


$$
\frac{d x}{d t}=v
$$

and Newton's second law for the particle velocity:

$$
m \frac{d v}{d t}=F,
$$

where $\mathrm{F}$ represents the forces on the particle including body forces and aerodynamic surface forces. ${ }^{12}$ Here, we have not considered gravitational forces.

\section{B. Visualization of Finite Time Lyapunov Exponent (FTLE) with MANGEN}

We use MANGEN to determine the contours of FTLEs. MANGEN requires as input a dataset that contains the velocity field data within a time interval. Here, we take the exported field data from the Fluent computation as this input.

MANGEN computes fluid particle trajectories of an initially uniformly seeded grid of fluid particles by integrating the kinematic equation

$$
\dot{x}=v(x, t),
$$

with fourth-order Runge-Kutta Fehlberg method. With the distance between neighboring particles, $\phi(t)$, the FTLE, $\sigma_{t_{0}}$ is determined with

$$
\sigma_{t_{0}}^{T}(x)=\frac{1}{|T|} \ln \left\|\frac{d \phi_{t_{0}}^{T+t_{0}}(x)}{d x}\right\| .
$$

The FTLE so determined monitors the divergence of neighbouring particles from a time $t_{0}$ to a time $t_{0}+T$.

The time interval $T$ should be chosen large enough for the FTLE contours to have converged to one contour at a given time. Since the square cylinder flow is (nearly) periodic, the determination of the FLTE with one time period of data should determine the FTLE accurately. We found that using three periods of data improved the visualization, in particular the sharpness of FTLE contour maxima, of the square cylinder flow. No significant improvement was observed by using a larger time interval.

Since the kinematic equation is linear the fluid particle trajectories and the FTLE may be determined in backward or forward time. In forward time, the FTLE thus identifies divergent stretching of the fluid field and maxima of the FTLE identify repelling transport barriers in the flow, better known as Lagrangian Coherent Structures. In backward time, the FTLE contours visualizes the convergent stretching or shrinking of fluid particles and maxima on the backward-time FTLE contours identify the attracting transport barriers in the flow.

\section{Computational Setup for the Square Cylinder Flow Simulation}
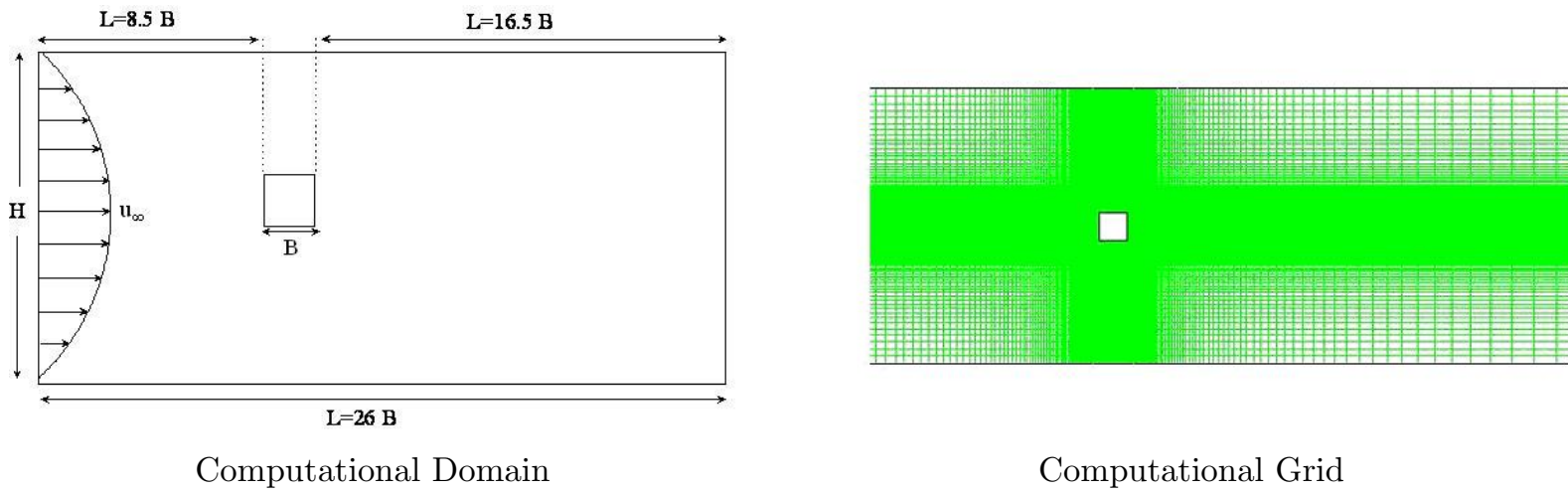

Computational Grid

Figure 1. Schematic of computational domain and grid for computation of the flow over a $10 \%$ confined square placed in a channel at a Reynolds number of $R e=150$. 
In Fig. 1, we show the computational domain used for the two-dimensional flow simulations over the square cylinder. The non-dimensional width of the square cylinder is equal to unity, $B=1$. Following the detailed domain dimension and grid study in Ref. 11, the cylinder is placed in a channel with a total length, $L=26 B$. The leading edge of the cylinder is located $8.5 B$ units from the inlet ensuring negligible boundary effects. The height of the channel, $H$ determines the blockage, $B / H$, of the cylinder by the channel. We have considered a $10 \%$ and a $33 \%$ blockage.

We take the Reynolds number of the flow based on the square width and the average inflow velocity, $R e=U_{f} L_{f} / \nu=150$. Here, $\nu$ is taken the viscosity of air at room temperature. A parabolic velocity profile, with a mean non-dimensional streamwise velocity equal to unity, is imposed at the inlet and the "outflow" boundary condition available in Fluent is specified at the outlet. A no-slip boundary condition is imposed at the top and bottom walls of the channel.

The grid is generated following the detailed grid independence study by SE. A block structured grid meshes the computational domain. In the area immediately around the cylinder, the grid is refined to capture the large velocity gradients that occur in that region, while a coarser grid is used upstream and downstream of the cylinder. The first cell neighbouring the cylinder is 0.01 units high. The grid is refined in the wake of the cylinder to ensure sufficient resolution of unsteady Karman street. For B/H $=10 \%$, a grid size of $323 \times 300$ is used. For $\mathrm{B} / \mathrm{H}=33.3 \%$, a grid sizes of $323 \times 334$ was used. A gradient was used to ensure that the finest grid spacing occurred near the cylinder. The grid that converged the $10 \%$ confined flow case and yielded excellent comparison within $1 \%$ of the drag coefficient, the Strouhal number and the recirculation length of the dead water region in the average flow field as reported by SE is shown in Fig. ??. The computational domain and grid of the $33 \%$ confined case is identical except for a scaling in the channel wall normal direction. The time step chosen for this simulation is $\Delta t=0.005$ and corresponds to the time step used by SE.

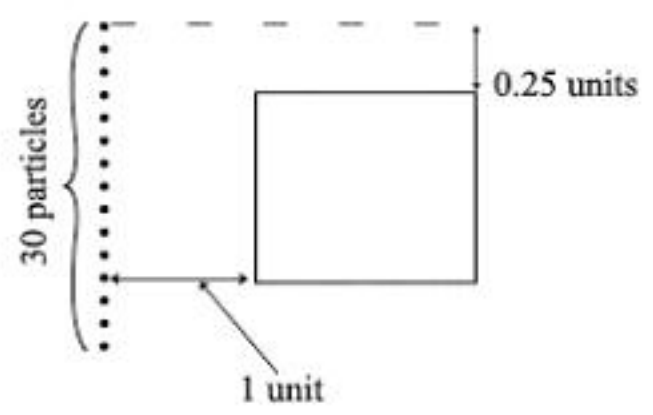

Figure 2. Schematic of particle injection locations.

To study the relation between particle transport and the LCS, we periodically inject thirty particles upstream from the cylinder as shown in Fig. 2. We consider fluid particles and finite sized particles with three Stokes numbers, $S t=t_{d} / t_{f}=0.1,1$, and 6.9. The characteristic flow time is defined as $t_{f}=L_{f} / U_{f}$, where $U_{f}$ is taken as the bulk inflow velocity. We take the ratio of the particle to fluid density one thousand. In this case, the predominant force on the particle is the Stokes drag.

\section{Results and Discussion}

By comparing the computationally determined traces of individual particles to FTLE contours, we shed light on the entrainment mechanisms of the particles into the near wake and the far wake. Snapshots of the particles superimposed on the FTLE contours are used to visualize the particle dispersion as compared to the Lagrangian Coherent Structures.

Contours of the FTLE determined in forward time identify repelling transport barriers in the flow field over the square cylinder in Fig. 3a and b. These repelling barriers form enclosed lobes in front of the square in which particles are trapped, much like the lobes described in the propulsion of jellyfish in Ref. 10. As the flow evolves, the lobe and the particles enclosed by the lobe are convected downstream and to the side of the cylinder (Fig. 3b). The lobe transports the fluid particles further to the rear of the cylinder into the 
time-periodic unsteady wake. Contours of the backward FTLE visualize the vortices in the near wake (Fig. $3 \mathrm{c})$.

Attracting fluid transport barriers as identified by the maxima of the backward FTLE entrain the particles into the recirculation region behind the square through a jet that forms between the two LCS that periodically grow and shed into the far wake. From this jet location, the fluid particles are transported throughout the unsteady vortex system on the side and rear of the square. The FTLE in the jet does not provide the information as to the future location of the particle around the square. The fluid particle may end up in either one of the large LCS on the rear, or any other of the smaller LCS on the side and rear.

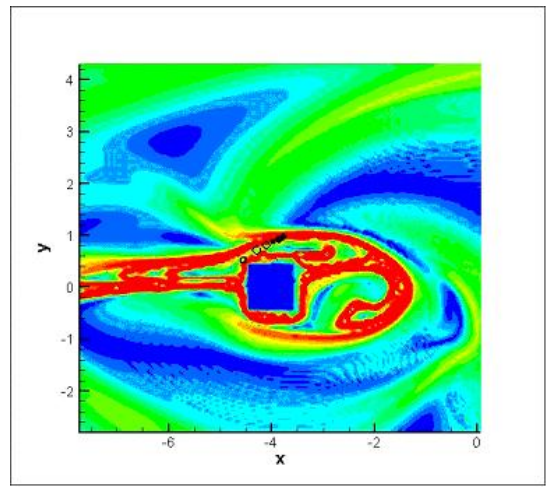

(a) Repelling FTLE

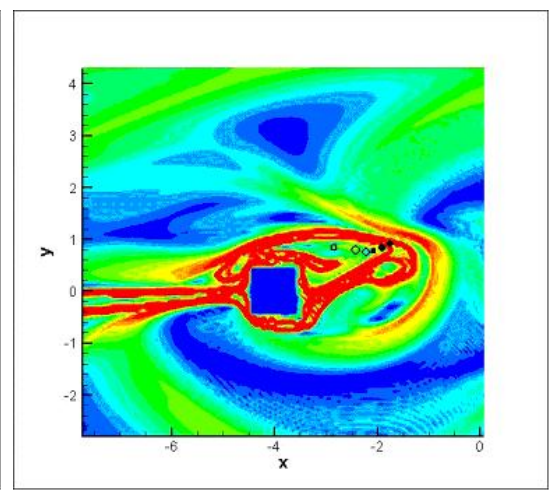

(b) Repelling FTLE at later time

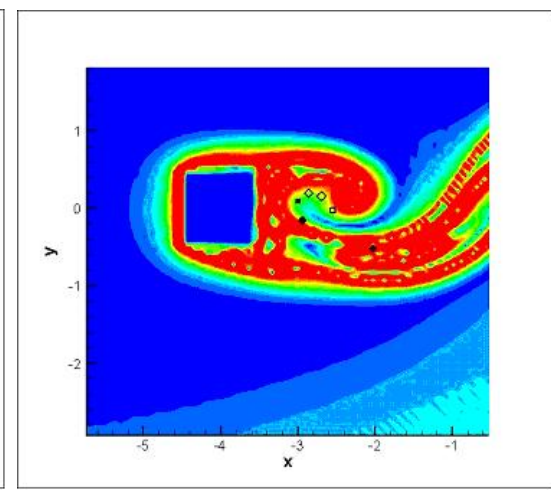

(c) Attracting FTLE

Figure 3. Visualizations of the LCS and fluid particles near the square cylinder. Maxima of the forward FTLE identify repelling transport barriers in the flow, that form lobes ahead of the square (a). The lobes and the particles enclosed by the lobes are convected downstream(b). Maxima of the backward FTLE (c) identify attracting transport barriers, that entrain the particles in the near weak behind the square.

The two large structures that formed at the rear of the cylinder alternately shed and convect downstream into the wake of the cylinder forming the LCS topology as shown in Fig. 4a: An alternating pattern of structures enclosed by the backward time FTLE barrier, are connected by a curved transport barrier. Fluid particles that are entrained into the two large structures behind the square are trapped into these alternating FTLE enclosures. Other fluid particles that were not entrained are attracted to the backward time FTLE, and therefore increasingly align downstream in the wake with the attracting transport barrier.

At $S t=0.1$ the particle response to the fluid is relatively fast, and the dispersion of the particles compares closely to the fluid particle dispersion (Fig. 4b). However, even at this low Stokes number particles are not entrained into the shedding vortices like the fluid particles. The inertial particles do not follow the trajectory into the jet between the two large structures behind the square. Inertial forces, even though small in magnitude, and the resulting delayed response to the carrier fluid, prevent the finite sized particles to make the sharp turn that the fluid particles make into the jet and the near wake. Thus, even at this low Stokes number, inertial particles do not entrain into the large structures. As a result, no particles are observed at the center of the enclosed FTLE structures in the far wake.

At $S t=1$ the particles dispersion displays the particle focusing (Fig. 4c) discussed in Ref. 5, i.e. the particles align in sharp material lines. The focusing was attributed to the the radial centrifugation of particles induced by the vortices as identified by the vorticity contours. Comparison of the particles dispersion to the flow topology visualized with the FTLE, puts particle focusing in a new light. We observe that part of the particle lines are in exact alignment with the fluid attractors that connect the large structures. When the curvature of these barriers increases, the inertial particles decreasingly follow the barriers and at some point break away from them. When the separated inertial particles line encounters the enclosed vortex structure in the far wake, the particle stagnates and consequently moves around the front of the structure. Clearly, the attractor on the edges of the vortex enclosure in the far wake is strong, consistent with the concentrated circulation that is generated in the FTLE enclosure when it grows and consequently sheds at the rear of the square.

If the particle inertia further increases at higher Stokes numbers, the inertial particles follow the carrier fluid to an even lesser extent and the inertial particle then show a lesser correlation with FTLE. However, if the particle response time is equal to the shedding period, i.e $S t=6.9$, the particles entrain into the gaps 


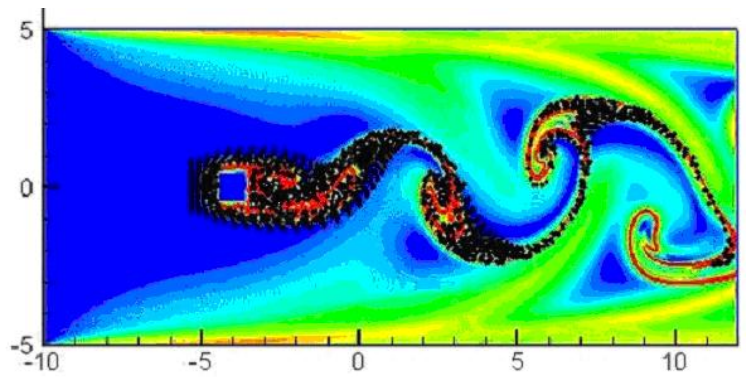

(a) Fluid

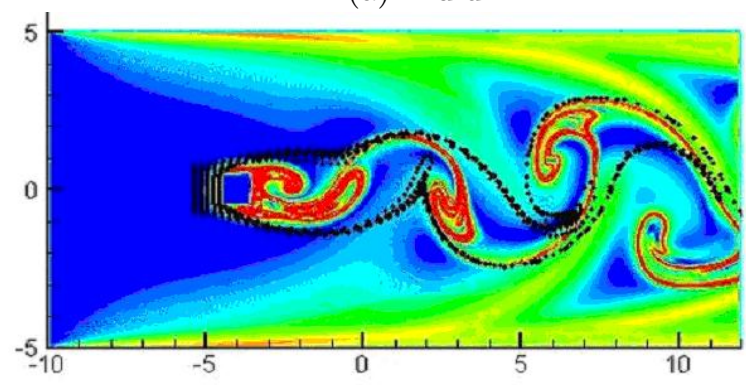

(c) $S t=1$

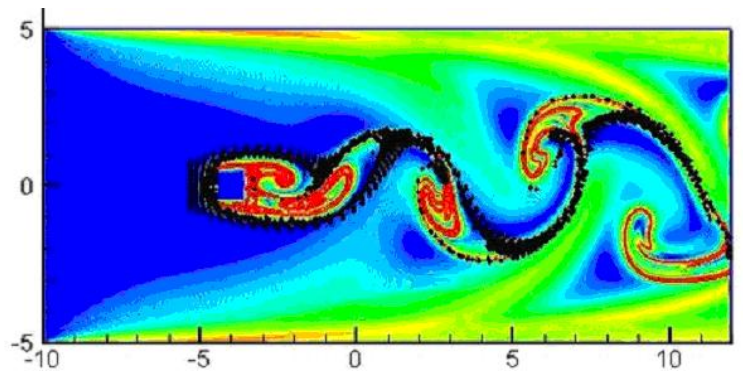

(b) $S t=0.1$

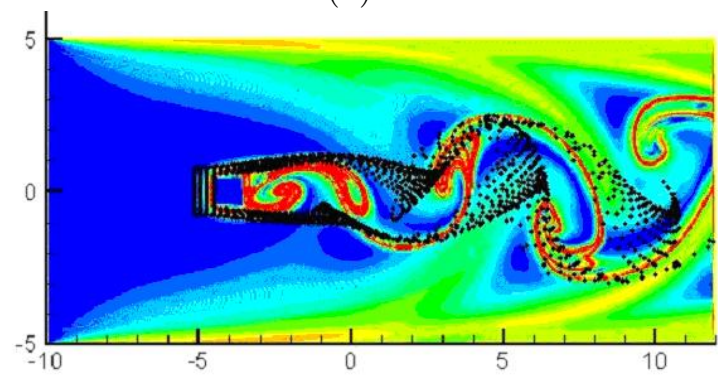

(d) $S t=6.9$

Figure 4. Snapshots of fluid particles (a) and inertial particles with $S t=0.1$ (b), $S t=1$ (c), and $S t=6.9$ (d) and the contours of the FTLE determined in backward time visualizing the LCS in the flow over a $10 \%$ confined square cylinder. 
between the vortex enclosures in the far wake, and consequently accumulate at the rear of these vortices as described in Ref. 8.
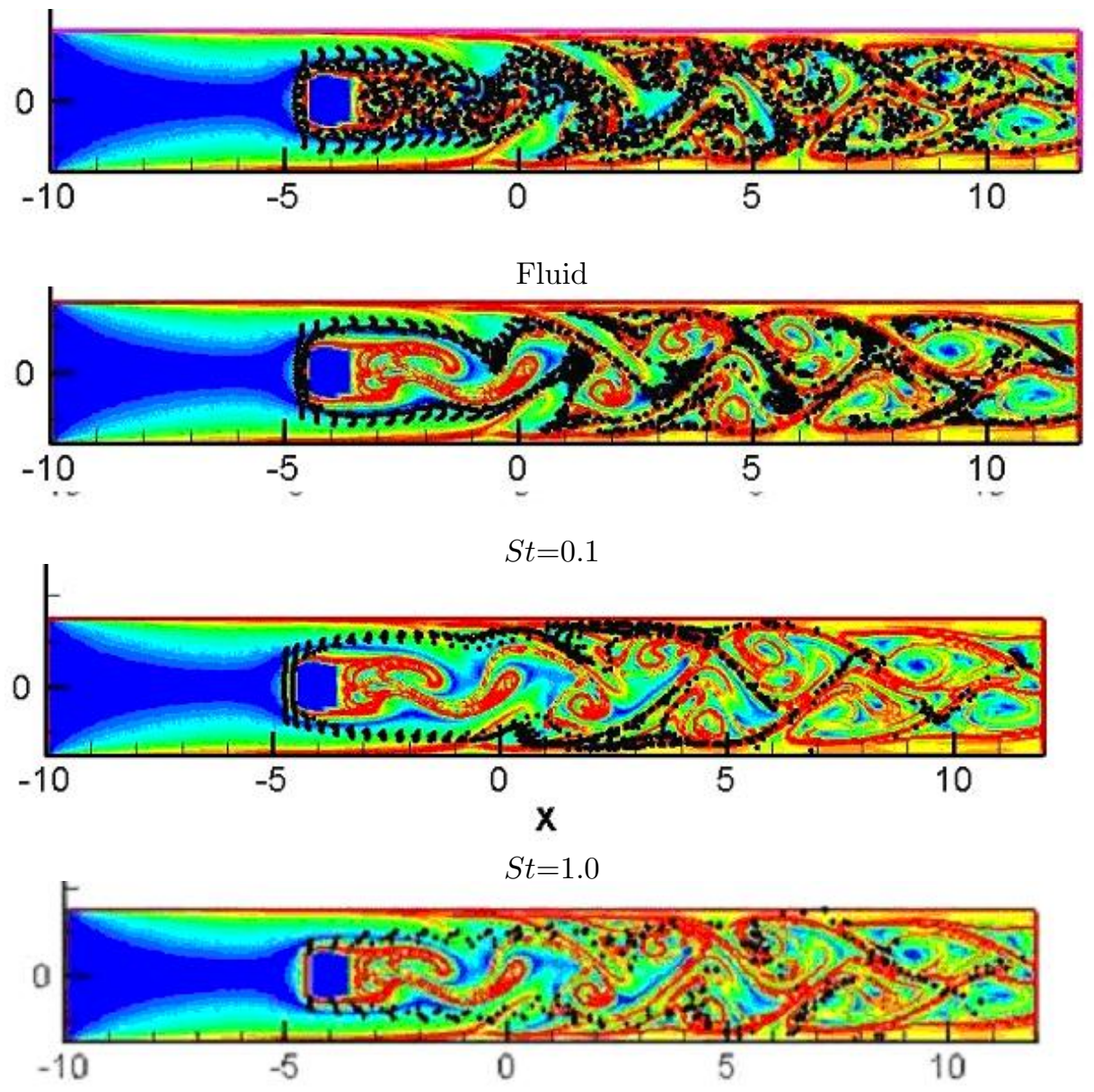

$S t=6.0$

Figure 5. Same as Fig. 4, but for a $30 \%$ confined particle-laden flow over the square.

With increased blockage the vortices in the near wake of the cylinder flattens and elongates (Fig. 5). This leads to an entrainment of the fluid particles at locations further downstream of the cylinder. The mechanism, however, is the same as described for the lower blockage case. Moreover, FTLEs that originate at the channel walls now significantly affect the structures downstream in the channel transporting fluid particles towards the center of the channel.

Inertial particles show a comparable deviation to the fluid particles as for the $10 \%$ confined case. At $S t=0.1$ particles are not entrained in the near wake. At $S t=1$ the particles align along the FTLEs, whereas at $S t=6$, particles accumulate in packages and closely follow the LCS.

\section{Conclusion}

We have investigated the relation between inertial particle transport and fluid particle transport as visualized by the Lagrangian transport barriers in the FTLE contours in the flow over a square cylinder placed in a channel.

Whereas fluid particles are entrained into the near wake by the lobe structures in front of the cylinder formed by repelling transport barriers in the forward-time FTLE field, inertial particles with relatively fast response times are not following the sharp turns that fluid particles make into the large attracting Lagrangian 
structure in the near wake. Inertial particles with $S t>0.1$ are not transported into the near wake.

Particles with Stokes number, $S t \leq 1$, are increasingly accumulating near the attracting transport barriers in the Lagrangina wake. At $S t=1$, when particles are well-known to exhibit particle focusing, large parts of the particle streak are fully aligned with transport barrier. The attracting LCS in the wake is hence excellent predictor of the location where particles focus.

With increasing blockage separated transport barriers that form on the wall and reach into the flow that transport fluid and inertial particles towards the center of the channel. The separated barriers interact with the Lagrangian wake behind the square and enhance mixing of the particles.

\section{Acknowledgments}

The first author appreciates the support by the University Grants Program at San Diego State University.

\section{References}

${ }^{1}$ Haller, G., "Finding Finite-Time Invariant Manifolds in Two-Dimensional Velocity Fields," Chaos, Vol. 10, No. 1, 2000, pp. $99-108$

${ }^{2}$ Haller, G. "Lagrangian Coherent Structures from Approximate Velocity Data", Phys. Fluids, Vol. 14, 2002, pp. $1851-1861$.

${ }^{3}$ Haller, G., "An Objective Definition of a Vortex," Journal of Fluid Mechanics, Vol. 525, 2005, pp. 1-26.

${ }^{4}$ Crowe, C.T., Gore, R.A., and Troutt, T.R., "Particle Dispersion by Coherent Structures in Free Shear Flows," Particle Science and Technology, Vol. 3, 1985, pp. 149-158.

${ }^{5}$ Tang, L., Wen, R., Yang, Y, Crowe, C.T., Chung, J.N. and Troutt, T.,R., "Self-Organizing Particle Mechanism in a Plane Wake," Physics of Fluids A, Vol. 4, No. 10, 1992, pp. 2244-2251.

${ }^{6}$ Sohankar, A., Norberg, C. and Davidson, L., "Low-Reynolds-Number Flow around a Square Cylinder at Incidence: Study of Blockage, Onset of Vortex Shedding and Outlet Boundary Condition," International Journal for Numerical Methods in Fluids, Vol 26, No 1, 1998, pp. 39-56.

${ }^{7}$ Brandon, D.J., and Aggarwal, S.K., "A Numerical Investigation of Particle Deposition on a Square Cylinder Placed in a Channel Flow," Aerosol Science and Technology, Vol. 34, No. 4, 2001, pp. 340-352.

${ }^{8}$ Jacobs, G.B., Kopriva, D.A., and Mashayek, F., "Compressible Subsonic Particle-Laden Flow over a Square Cylinder," Journal of Propulsion and Power, Vol. 20, No. 2, 2004, pp. 353-359.

${ }^{9}$ Mathur, M., Haller, G., Peacock, P., Rupper-Felsot, J.E., and Swinney, "Uncovering the Lagrangian Skeleton of Turbulence", Phys. Rev. Lett., Vol. 98, 2007.

${ }^{10}$ Shadden, S.C, Dabiri, John. O., and Marsden, Jerrold, E., "Lagrangian Analysis of Fluid Transport in Empirical Vortex Ring Flows", Phys. Fluids, Vol. 18, 2006, pp. 105-111.

${ }^{11}$ Sharma, A. and Eswaran, V., "Effect of Channel Confinement on the Two-Dimensional Laminar Flow and Heat Transfer Across a Square Cylinder", Numerical Heat Transfer, Part A, Vol 47, 2005, pp. 79-107.

12 Maxey, M.R. and Riley, J.J., "Equation of Motion for a Small Rigid Sphere in a Non-Uniform Flow", Physics of Fluids, Vol. 26, 1983, pp. 883-889. 